\title{
PRODUCTION ACADÉMIQUE ET ÉDUCATIVE D'UN ÉTUDIANT À LA MAÎTRISE EN EPT OF IFAP MACAPÁ, AMAPÁ, AMAZÔNIA, BRÉSIL
}

\section{ARTICLE ORIGINAL}

VIANA, Valderi Nascimento ${ }^{1}$, FECURY, Amanda Alves², OLIVEIRA, Euzébio de ${ }^{3}$, DENDASCK, Carla Viana ${ }^{4}$, DIAS, Claudio Alberto Gellis de Mattos ${ }^{5}$

VIANA, Valderi Nascimento. Et al. Production académique et éducative d'un étudiant à la maîtrise en EPT of IFAP Macapá, Amapá, Amazônia, Brésil. Revista Científica Multidisciplinar Núcleo do Conhecimento. An 06, Ed. 06, Vol. 08, p. 186 à 200. Juin 2021. ISSN: 2448-0959, Lien d'accès: https://www.nucleodoconhecimento.com.br/education-fr/etudiant-a-la-maitrise, DOI: 10.32749/nucleodoconhecimento.com.br/education-fr/etudiant-a-la-maitrise

\section{RÉSUMÉ}

La production scientifique dans les cours de premier cycle et d'études supérieures a son importance pour la construction d'une nouvelle façon de penser de l'étudiant. Le Master professionnel en formation professionnelle en réseau national (ProfEPT) réunit le contenu disciplinaire, les connaissances pédagogiques et la production académique finale et les relie à des situations réelles de pratique éducative, et le travail final peut être présenté de différentes manières (articles, livres, produits éducatifs). La production académique, tant du conseiller que du guide, en plus de l'importance liée à

\footnotetext{
${ }^{1}$ Éducateur physique, spécialiste en physiologie de l'exercice et nutrition sportive (FAMA), étudiant du programme d'études supérieures en éducation professionnelle et technologique (PROFEPT IFAP).

${ }^{2}$ Biomédical, PhD en maladies tropicales, professeur et chercheur du cours médical du campus de Macapá, Université fédérale d'Amapá (UNIFAP).

${ }^{3}$ Biologiste, docteur en maladies tropicales, professeur et chercheur du cours d'éducation physique de I'Université fédérale du Pará (UFPA).

${ }^{4}$ Théologien, Ph.D. en psychanalyse clinique. II travaille depuis 15 ans avec la méthodologie scientifique (méthode de recherche) dans le cadre de l'orientation de production scientifique des étudiants à la maîtrise et au doctorat. Spécialiste de l'étude de marché et de la recherche axée sur la santé. Doctorant en Communication et Sémiotique (PUC SP).

${ }^{5}$ Biologiste, Ph.D. en recherche théorique et comportementale, professeur et chercheur du cours de chimie de I'Institut d'éducation fondamentale, technique et technologique de l'Amapá (IFAP) et du Graduate Program in Professional and Technological Education (PROFEPT IFAP).

RC: 89148
}

Lien d'accès: https://www.nucleodoconhecimento.com.br/education-fr/etudiant-a-la-maitrise 
la diffusion scientifique, a du poids dans l'évaluation du programme et aussi dans son maintien. L'objectif de ce travail est de montrer quantitativement la production académique et éducative d'un étudiant à la maîtrise en enseignement professionnel et technologique (EPT) de l'Institut d'éducation, de science et de technologie (PIPT) de Macapá, Amapá, Amazônia, Brésil. Au cours de la maîtrise, la production scientifique sous diverses formes s'est avérée nécessaire au cours du processus de formation. L'union de la théorie avec la pratique de l'écriture, a facilité la production de la thèse qui devrait être présentée à la fin du cours. Grâce au processus d'organisation, la planification des idées a abouti à une nouvelle pensée structurée selon les normes de l'académie, mais cela ne se limite pas à cet espace et à ces normes. La production pendant la maîtrise vise à contribuer à la fois quantitativement et qualitativement à la production scientifique de la région nord, en l'impactant positivement sur celle-ci et le programme de maîtrise. En plus de l'importance de la contribution de la création et de la diffusion scientifiques, les productions ont tendance à avoir un impact positif sur l'évaluation et le maintien du programme PROFEPT-PIPT, réalisé par CAPES.

Mots-clés : EPT, Diffusion, Production scientifique, PRORFEPT.

\section{INTRODUCTION}

Unissant les instruments théoriques et méthodologiques opportunités la réalisation de nouveaux projets, l'étudiant cesse d'être juste un répétiteur et commence à produire des connaissances, contribuant avec un thème ou un domaine spécifique. Par la construction de sa propre pensée, ils produisent de nouvelles attitudes et de nouveaux comportements, c'est-à-dire qu'ils exposent une idée plausible et structurée, présentant des jugements de valeur et un sens critique avec une argumentation valide et logique (Maia, 2008).

Le Master professionnel en formation professionnelle en réseau national (ProfEPT) apparaît comme une opportunité de formation spécifique sur l'enseignement professionnel et technologique pour les employés des instituts fédéraux et la population externe, ayant la production de connaissances à travers des produits 
éducatifs et des réalisations de recherche qui se rapportent au monde du travail. Dans cette modalité, l'objectif est l'union du contenu disciplinaire et des connaissances pédagogiques, la production académique finale doit être liée à des situations réelles de pratique éducative, et le travail final peut être présenté de différentes manières (Machado e Urbanetz, 2019; Urbanetz et al., 2020).

Pour la production et la publication de connaissances scientifiques, l'orientation joue un rôle fondamental. Le conseiller ayant une vaste expérience dans l'édition, devient un mentor pour son mentorat, ayant un impact positif sur la productivité, étant responsable des progrès d'apprentissage et de la réussite dans l'avenir de l'étudiant. C'est-à-dire que toute l'expérience acquise au cours du processus de formation a un impact positif sur la vie académique de l'étudiant, les compétences et les compétences pour la publication sont apprises au cours de la formation (Falaster et al., 2017).

Selon le formulaire d'évaluation des programmes académiques et professionnels (Domaine 46: Enseignement) de la Coordination pour l'amélioration du personnel de l'enseignement supérieur (CAPES):

[...] la capacité de production minimale pertinente de sera évaluée et peut impliquer des étudiants et des diplômés. De plus, l'adhérence et l'alignement de la production indiquée par le DP ppg (quatre productions) avec sa zone de concentration et ses lignes de recherche seront évalués, reflétant la cohérence de la production avec les objectifs et l'identité du programme. [...] Pour cela, le PPG indiquera jusqu'à cinq productions bibliographiques et jusqu'à cinq PTT publiées au cours de la période de quatre ans impliquant des étudiants et des diplômés. [...] la pertinence de la production indiquée par rapport à l'impact et au caractère innovant de la production intellectuelle, de la couverture locale, régionale, nationale ou internationale et de la satisfaction des exigences sociales. (Capes, 2020).

La production académique, tant du conseiller que du mentor, en plus de l'importance liée à la diffusion scientifique, a du poids dans l'évaluation du programme d'études supérieures stricto sensu et aussi dans son maintien. 


\section{OBJECTIF}

Démontrer quantitativement la production académique et éducative d'un étudiant à la maîtrise en enseignement professionnel et technologique (EPT) de l'Institut d'éducation, de science et de technologie (IFAP) de Macapá, Amapá, Amazônia, Brésil.

\section{MÉTHODOLOGIE}

Cet article expose la production intellectuelle développée au cours du ProfEPT - IFAP. Ces productions qui peuvent être classées comme production bibliographique (article dans un périodique, livre) et production technique (application - APP, site web)

Les enquêtes quantitatives visant à obtenir des données sur l'application et le site Web ont utilisé la période du 12 avril 2021 au 14 juin 2021. Les données relatives à l'application proviennent de la Play Console (Playconsole, 2021) et du site Web de Google Analytics (Googleanalytics, 2021).

Pour les livres et les articles scientifiques, la date de publication originale a été utilisée jusqu'au 14 juin 2021. Les données les concernant ont été tirées des donnéeson-line (ivres et articles publiés dans Revista Multidisciplinar Núcleo do Conhecimento) (Rmnc, 2021) ainsi que par consultation directe de la revue (article publié dans Research, Society and Development journal) (Rsd, 2021).

\section{RÉSULTATS}

\section{APPLICATION EFE (EDUFISEPT)}

La figure 1 montre le pourcentage d'installations d'applications EFE par source de trafic d'informations entre le 12 avril et le 14 juin 2021. Les données montrent que $21,67 \%$ (13 utilisateurs) sont arrivés à l'application par recherche dans le Google Play Store et $78,30 \%$ (47 utilisateurs) via un lien tiers. 
Figure 1 - indique le pourcentage d'installations d'applications EFE par source de trafic d'informations entre le 12 avril et le 14 juin 2021.

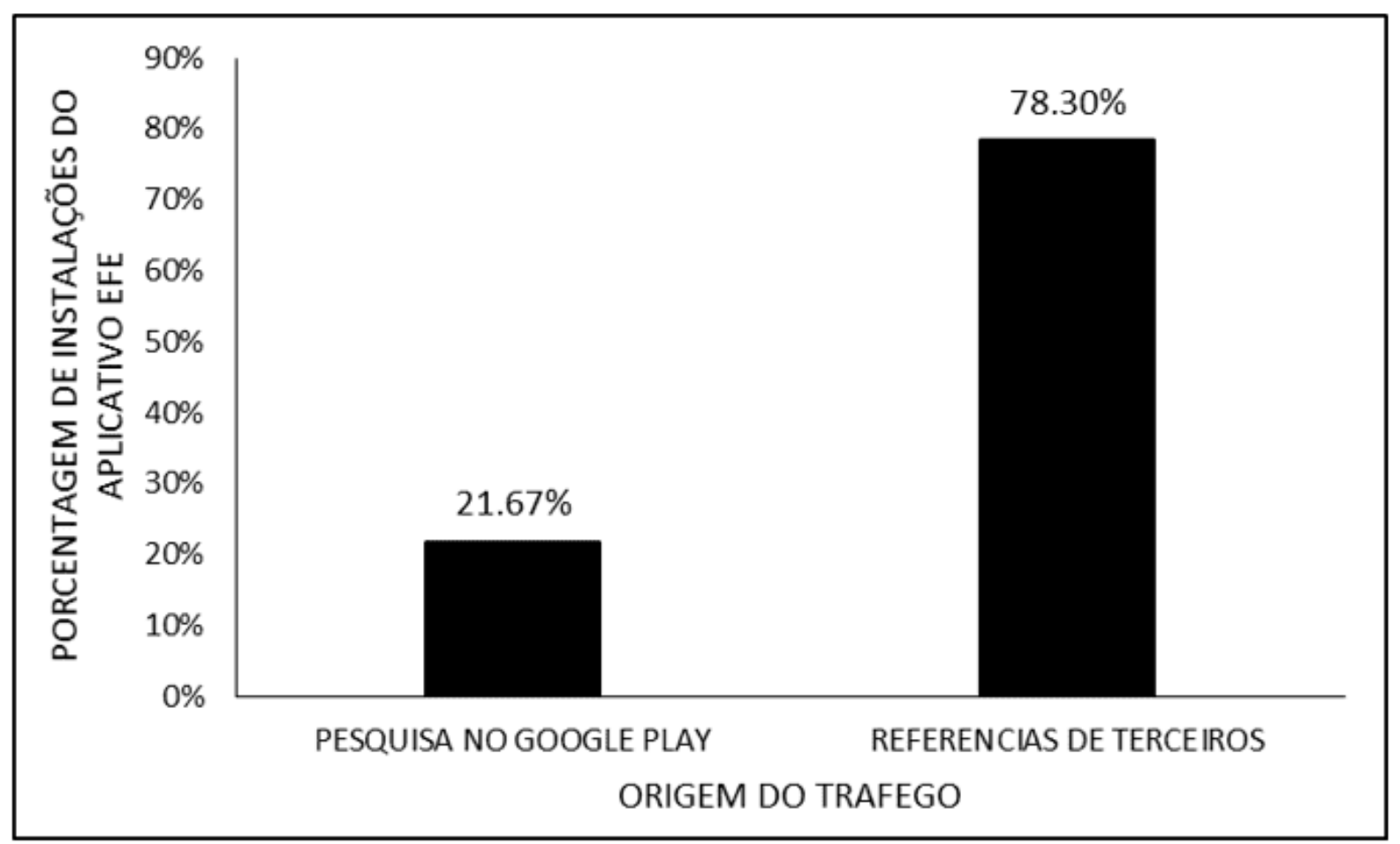

Source : Adapté de Playconsole (2021)

En ce qui concerne l'analyse du pourcentage d'installations de l'application EFE par pays de l'utilisateur, entre le 12 avril et le 14 juin 2021, il y a eu un record de téléchargements dans deux pays, soit 98,33\% (59 downloads) du Brésil et 1,67\% du Portugal (01d ownload) (Figure 2). 
Figure 2 - indique le pourcentage d'installations d'applications EFE par pays utilisateur entre le 12 avril et le 14 juin 2021.

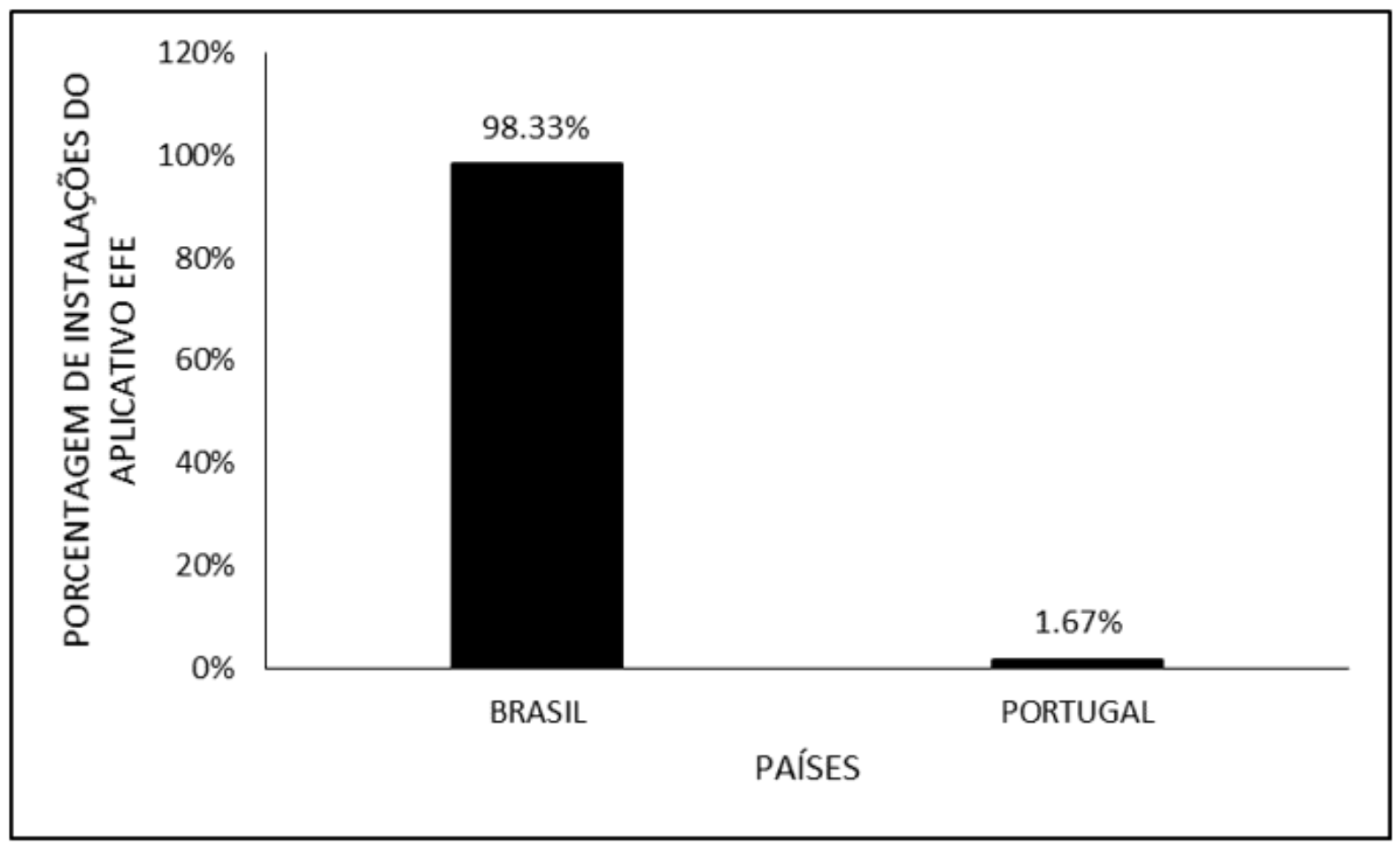

Source : Adapté de Playconsole (2021)

Le pourcentage d'installations d'applications EFE par visite et installation d'applications entre le 12 avril et le 14 juin 2021 est illustré à la figure 3 . Sur $100 \%$ des visiteurs de la page en magasin (82 personnes), $73,17 \%$ (60) personnes) ont téléchargé l'application. à leur appareil mobile (Figure 3). 
Figure 3 - Affiche le pourcentage d'installations d'applications EFE par visite et installation d'applications, entre le 12 avril et le 14 juin 2021.

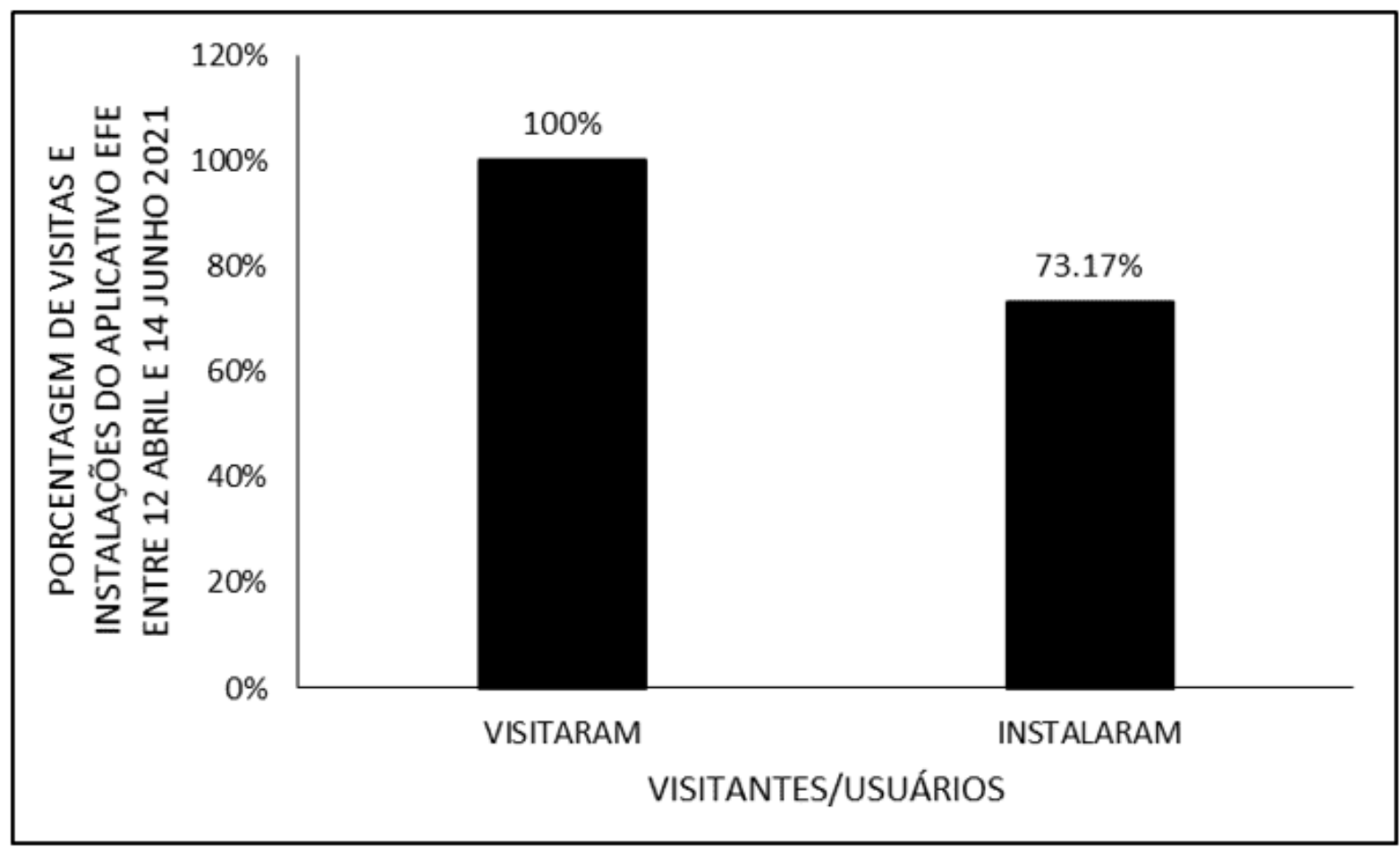

Source : Adapté de Playconsole (2021)

La figure 4 montre le pourcentage d'installations d'applications EFE par type d'utilisateur entre le 12 avril et le 14 juin 2021. Sur le total (60 utilisateurs), 93,33\% (56 personnes) sont de nouveaux utilisateurs et $6,67 \%$ (04 personnes) sont des utilisateurs récurrents au sein de l'application, c'est-à-dire ont accédé à l'APP plusieurs fois. 
Figure 4 - indique le pourcentage d'installations d'applications EFE par type d'utilisateur entre le 12 avril et le 14 juin 2021.

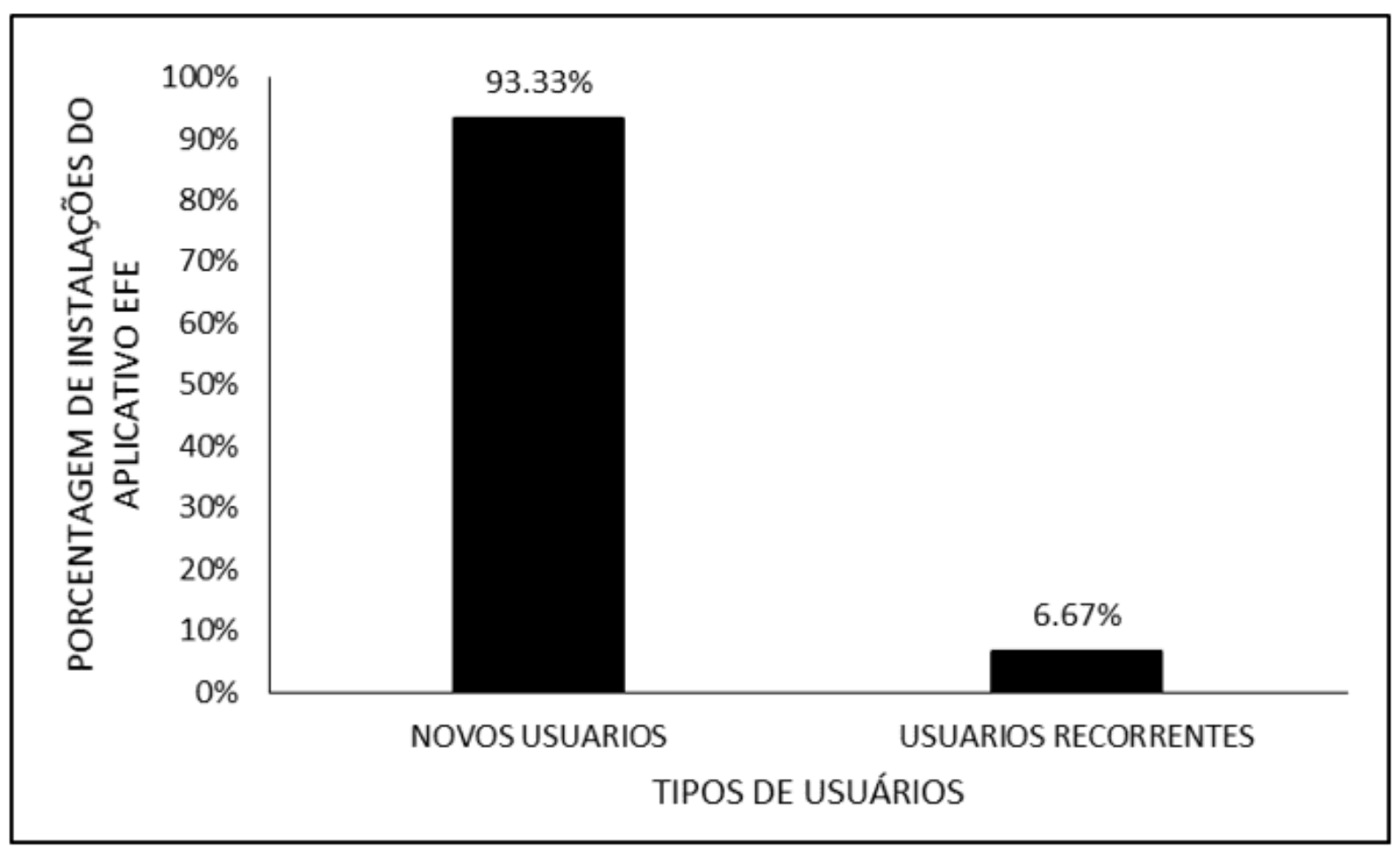

Source : Adapté de Playconsole (2021)

\section{SITE}

Concernant le pourcentage d'accès au site EduFisEPT par pages internes du site, entre le 12 avril et le 14 juin 2021, la page ayant le plus d'accès parmi les 1399 utilisateurs (100\%) est des pratiques pédagogiques avec 30,38\% (425 personnes). Vient ensuite la Page des sports avec 17,23\% (241 personnes accédant), les auteurs avec $13,37 \%$ (187 personnes), la collaboration avec 13,22 \% (185 personnes), les jeux et jeux avec 7,79\% (109 personnes accédant), les danses 5,65\% (79 personnes), la santé avec 5,00 \% (70 personnes), les loisirs avec 4,43\% (62 personnes) et les combats avec $2,91 \%$ (41 personnes) (figure 5 ). 
Figure 5 - Indique le pourcentage d'accès au site EduFisEPT par page interne entre le 12 avril et le 14 juin 2021.

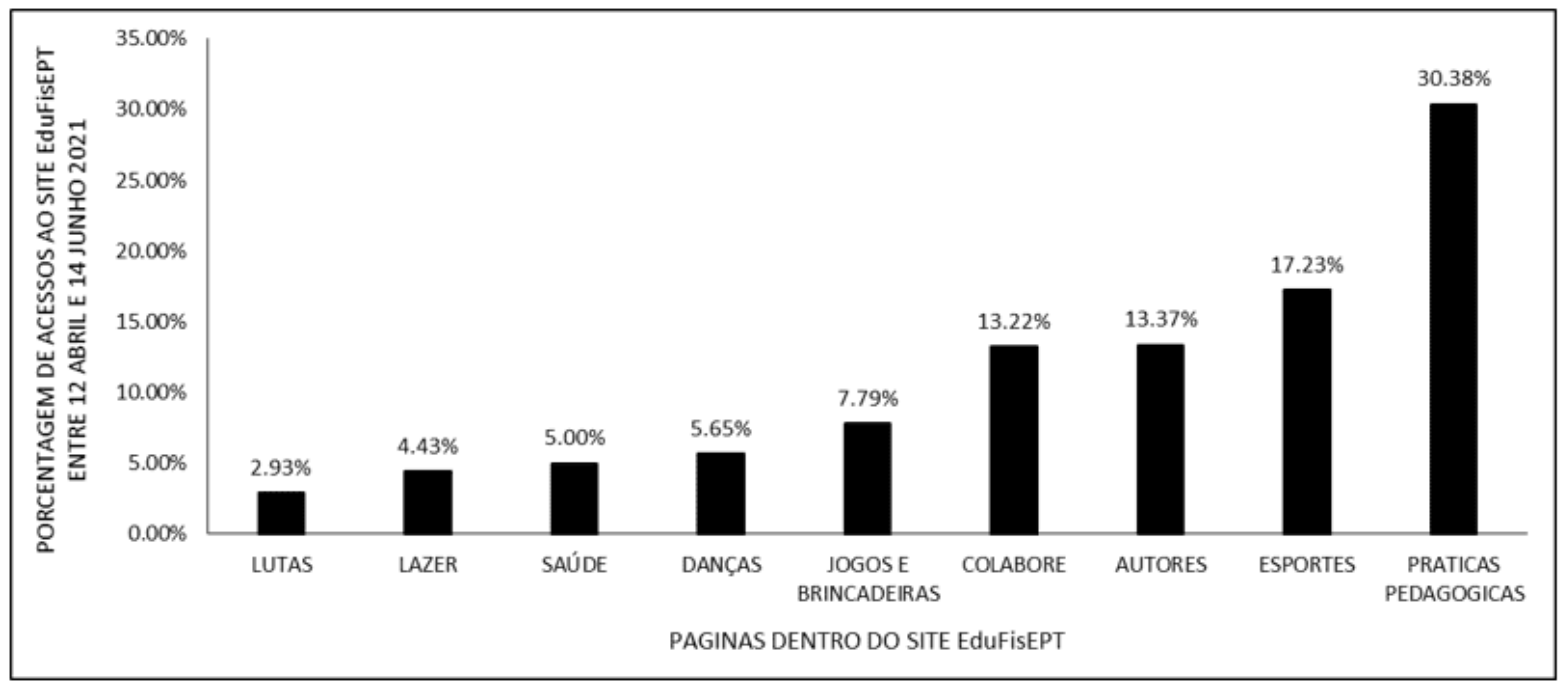

Source: Adapté de GoogleAnalytics (2021).

Le pourcentage d'accès au site EduFisEPT par pays, entre le 12 avril et le 14 juin 2021, porte le Brésil avec 93,92\% (1314 personnes) accès, suivi du Portugal avec 2,64\% (37 personnes), des États-Unis avec 1,50\% (21 personnes), du Mozambique $1,07 \%$ (15 personnes), 1,07\%, et de l'Irlande avec 0,86\% (12 personnes) (Figure 6). 
Figure 6 - Montre le pourcentage d'accès au site Web EduFisEPT par pays entre le 12 avril et le 14 juin 2021.

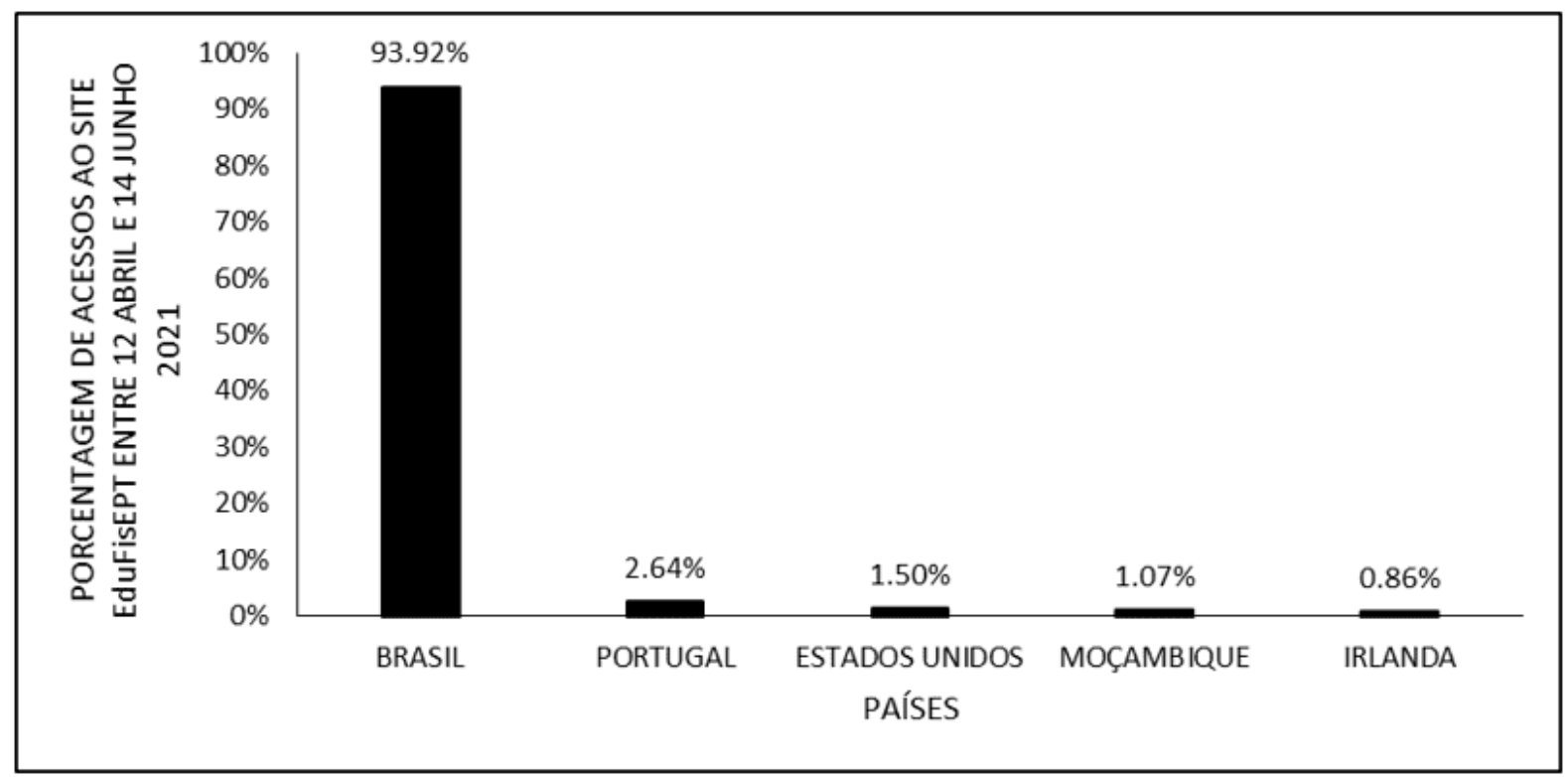

Source: Adapté de GoogleAnalytics (2021).

\section{LIVROS}

La figure 7 montre la couverture du livre publié en portugais (BR) le 30 septembre 2020. Depuis sa publication, le livre a cumulé un total de 715 vues. 
Figure 7 - Montre la couverture du livre publié en portugais (BR) le 30 septembre 2020.

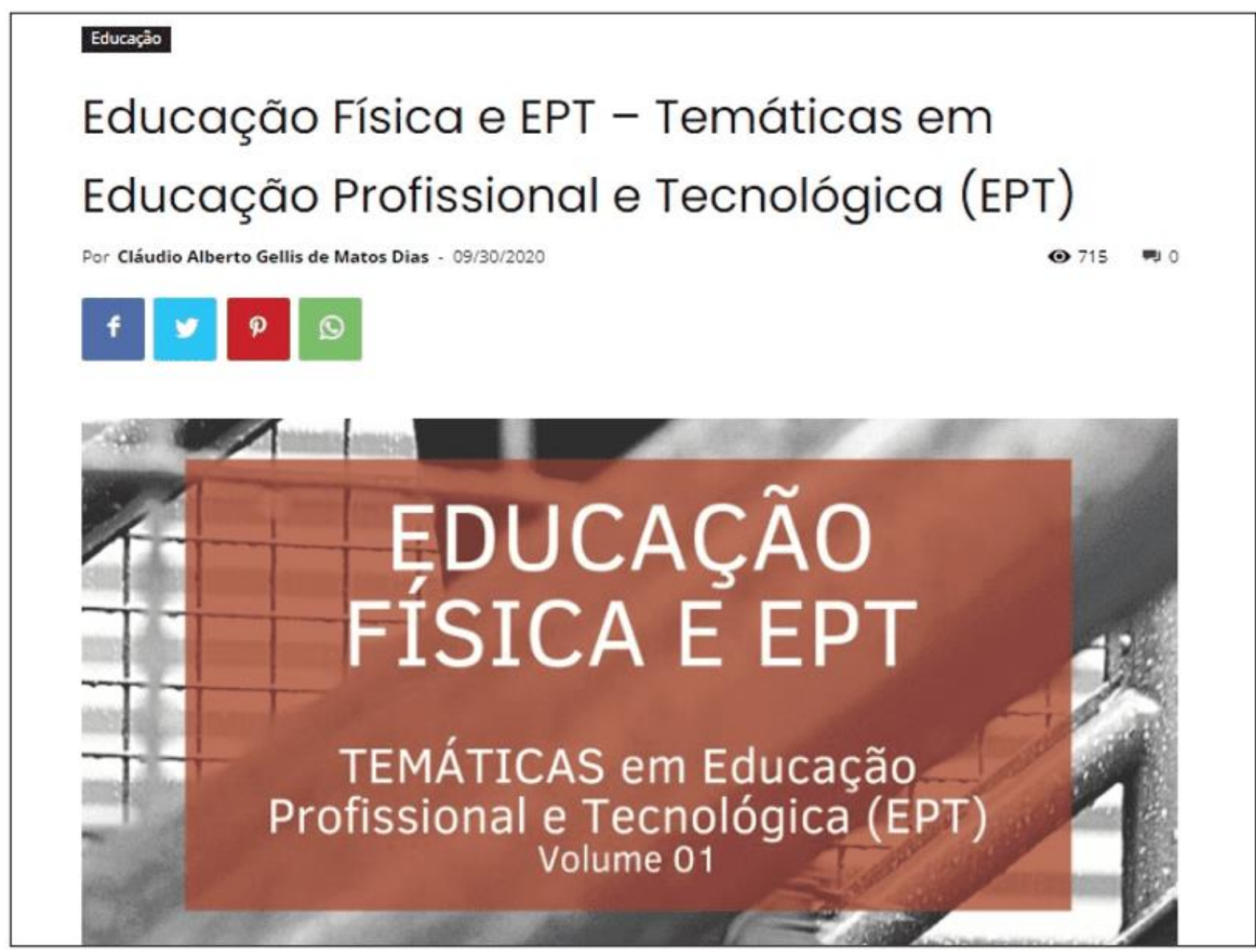

Source: Adapté de Viana et Dias (2020).

Le livre publié en anglais (version du livre en portugais) le 10 décembre 2020 cumule 248 vues depuis (Figure 8 ). 
Figure 8 - Montre la couverture du livre publié en anglais le 10 décembre 2020.

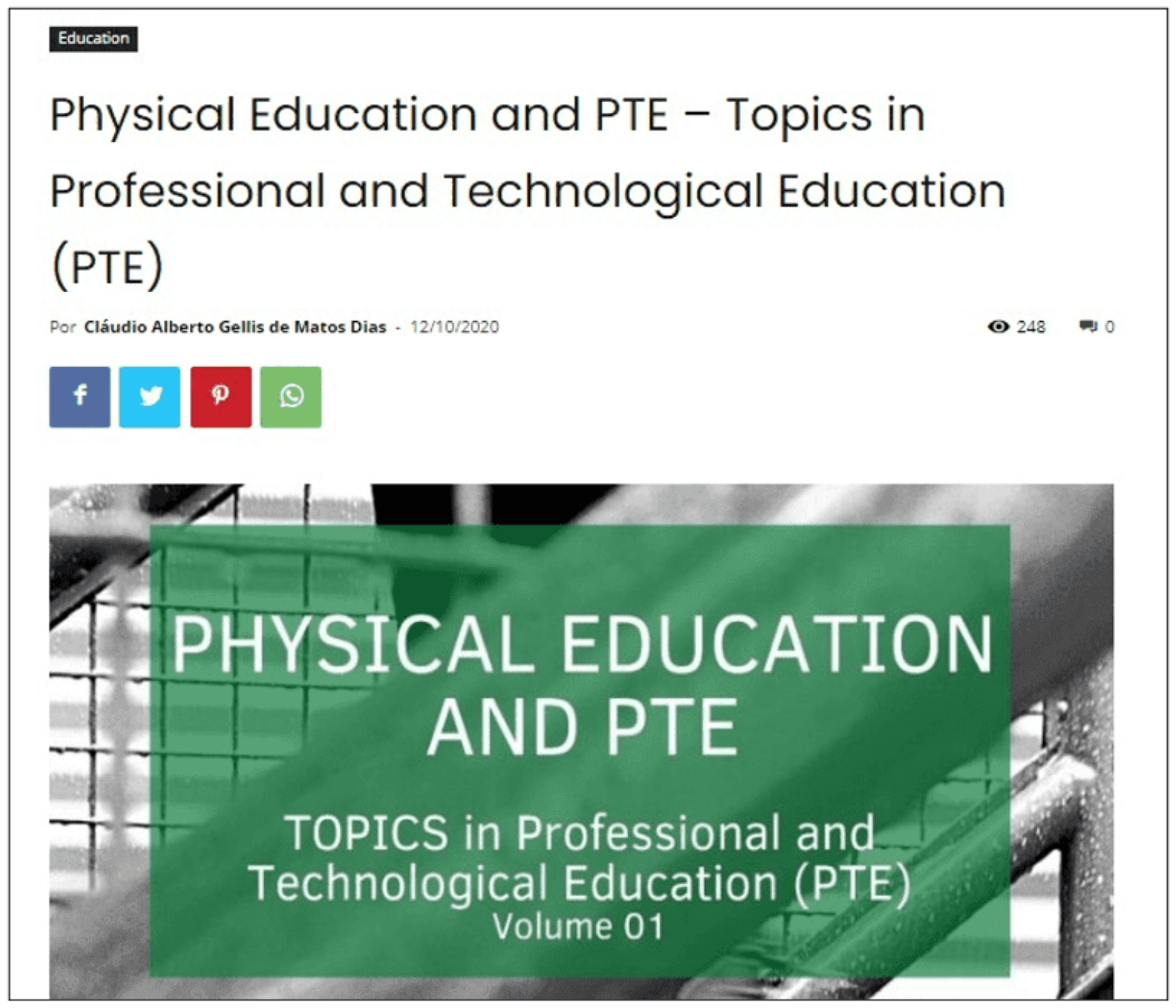

Source: Adapté de Viana et Dias (2020b).

\section{ARTICLES PUBLIÉS DANS DES REVUES INDEXÉES}

L'article publié dans la revue Research, Society and Development (Qualis 2013-2016 B2 et Qualis référence A3) le 2 juillet 2020 comptait, selon les informations des rédacteurs, 91 vues (Figure 9). 
Figure 9 - Montre la couverture de l'article publié dans la revue Research, Society and Development le 2 juillet 2020.

\begin{tabular}{|} 
Research, Society and Development, v. 9, n. 8, e239985704, 2020 \\
(CC BY 4.0) | ISSN 2525-3409 | DOI: http://dx.doi.org/10.33448/rsd-v9i8.5704 \\
Quadras de esportes em condições de uso adequado no Brasil: \\
influência no ensino-aprendizagem nas aulas de Educação Física (2015) \\
Sports courts in conditions of proper use in Brazil: influence on teaching-learning in \\
Physical Education classes (2015) \\
Canchas deportivas en condiciones de uso adecuado en Brasil: \\
influencia en la enseñanza-aprendizaje en las clases de Educación Física (2015) \\
Recebido: 16/06/2020 | Revisado: 18/06/2020 | Aceito: 22/06/2020 | Publicado: 02/07/2020 \\
Valderi Nascimento Viana \\
ORCD: https:/orcid.org/0000-0002-0885-5834 \\
Instituto Federal do Amapá, Brasil \\
E-mail: wallderinascimento@gmail.com
\end{tabular}

La figure 10 montre la couverture du manuel sur l'application EFE publié dans la Revista Multidisciplinar Núcleo do Conhecimento (référence Qualis B3) le 14 avril 2021. La publication en portugais a été vue par 244. 
La figure 10 montre la couverture du manuel sur l'application EFE publié dans la Revista Multidisciplinar Núcleo do Conhecimento le 14 avril 2021.

\section{Educaçâo Física}

MANUAL DE PRATICAS EM EDUCAÇÃO FÍSICA NA EDUCAÇÃO PROFISSIONAL E TECNOLÓGICA UTILIZANDO O APLICATIVO GRATUITO EFE - EPTFIS

Por Carla Dendasck - RC: 82194 - 14/04/2021

DOI: $10.32749 /$ nucleodoconhecimento.com.br/educacao-fisica/manual-de-praticas

\section{ARTIGO ORIGINAL}

VIANA, Valderi Nascimento [1], FECURY, Amanda Alves [2], OLIVEIRA, Euzébio de [3], DENDASCK, Carla Viana ${ }^{[4]}$, DIAS, Claudio Alberto Gellis de Mattos ${ }^{[5]}$

Source: Adapté de Viana et al. (2021).

Le manuel a été publié dans six autres langues, en plus de la langue portugaise. Le tableau 1 indique le nombre d'accès (et l'URL) au manuel sur l'application EFE publié dans la Revista Multidisciplinar Núcleo do Conhecimento jusqu'au 14 juin 2021, par langue. Au total, de la publication au 14 juin 2021, il y a eu 374 vues. 
Tableau 1 - Indique le nombre d'accès (et d'url) au manuel sur l'application EFE publié dans la Revista Multidisciplinar Núcleo do Conhecimento jusqu'au 14 juin 2021, par langue.

\begin{tabular}{|c|c|c|}
\hline IDIOMA & $\begin{array}{c}\text { NÚMERO } \\
\text { DE } \\
\text { ACESSOS }\end{array}$ & LINK DE ACESSO \\
\hline PORTUGUES & 244 & $\begin{array}{l}\text { https://www.nucleodoconhecimento.com.br/e } \\
\text { ducacao-fisica/manual-de-praticas }\end{array}$ \\
\hline INGLES & 20 & $\begin{array}{l}\text { https://www.nucleodocon hecimento.com.br/e } \\
\text { ducation-physics-en/manual-of-practices }\end{array}$ \\
\hline ESPANHOL & 24 & $\begin{array}{l}\text { https://www.nucleodoconhecimento.com.br/e } \\
\text { ducacion-fisica-es/manual-de-practicas }\end{array}$ \\
\hline FRANCÊS & 23 & $\begin{array}{l}\text { https://www.nucleodoconhecimento.com.br/e } \\
\text { ducation-physics-fr/manuel-de-pratiques }\end{array}$ \\
\hline ITALIANO & 26 & $\begin{array}{l}\text { https://www.nucleodoconhecimento.com.br/e } \\
\text { ducazione-fisica-it/manuale-di-pratiche }\end{array}$ \\
\hline ALEMÃO & 21 & $\begin{array}{l}\text { https://www.nucleodoconhecimento.com.br/b } \\
\text { ildung-physik-de/handbuch-der-praktiken }\end{array}$ \\
\hline RUSSO & 16 & $\begin{array}{l}\text { https://www.nucleodocon hecimento.com.br/o } \\
\text { бразование-физика-ru/пособие-по-практике, }\end{array}$ \\
\hline TOTAL & 374 & \\
\hline
\end{tabular}

Source : Adapté de Viana et al. (2021), Viana et al. (2021b), Viana et coll. (2021c), Viana et al. (2021d), Viana et al. (2021e), Viana et coll. (2021f), Viana et al. (2021g).

\section{DISCUSSION}

Dans l'environnement scientifique, toute la théorie et la méthodologie cherchent à rapprocher l'étudiant des connaissances produites par la science. Dans le cadre du processus de formation, le contact avec la rédaction d'articles, la construction de sites Web et l'application, la construction de la recherche, l'analyse et la diffusion des données, se sont avérés d'une grande importance. Dans chaque production, il y a une réalité qui fait l'objet de recherches, ce qui établit un lien avec les situations et les 
contextes socioculturels. La contextualisation des connaissances est un outil d'apprentissage significatif. II est opportuniste, nouvelle expérience ou connaissance résignification, est de développer la capacité d'analyser, hypothèses, sens critique au sein d'une structure actuelle, puisque l'interaction avec les faits et les phénomènes de la vie quotidienne se produit (Yamaguchi et Furtado, 2019).

Tous les documents publiés, physiquement ou en ligne, serventde moyen de diffusion scientifique. Dans le cas de l'application, tout le contenu couvert est basé sur des connaissances scientifiques, par un accès facile et simple. L'enseignant acquiert de nouvelles connaissances et peut les mettre en pratique au sein de l'école en temps réel. Selon Rossi (2019)

II reste à rapprocher la science des articles et des congrès de la vie des Brésiliens qui ont tant besoin de presque tout en matière de santé, de qualité de vie, de durabilité, de mobilité, d'utilisation de la technologie et de citoyenneté.

Ainsi, à la fois dans le niveau théorique (articles, livres, site Web) et l'application de l'application servent de stimulus à l'expérience scientifique, est de penser à la science articulée avec la réalité, l'accès et le langage accessible à tous ceux qui cherchent la connaissance. La diffusion va au-delà de la prise d'une connaissance systématisée, sert aussi à ravir, motiver et stimuler l'autre. (Rossi, 2019).

En éducation physique, la vision d'une pratique raffinée dans la technique par la technique ou la performance sportive est encore très forte. Le rendement et la technique doivent être pris en compte par l'enseignant, mais ne doivent pas être considérés comme des priorités et doivent respecter une norme d'excellence sans respecter l'individualité de l'élève. Dans une perspective de formation globale, les cours d'éducation physique doivent travailler leur objet d'étude dans une large dimension, puisque les élèves sont insérés dans une société qui a une culture et qui produit des significations, ainsi que révèle des contradictions de comportements, des attitudes qui doivent être abordées / considérées (Coutinho, 2010; Viana et al., 2020).

Ainsi, les productions scientifiques basées sur les sciences humaines et sociales ne contribuant qu'au domaine de l'éducation physique, dépassant uniquement l'utilisation 
des sciences de la santé, il est possible de placer l'éducation physique au même niveau de pertinence et d'importance que les autres composantes du programme scolaire (Balbino et Urt, 2018). Au cours du processus de recherche, des articles et des livres ont été traduits dans d'autres langues. Cette attitude est importante pour élargir la portée de la recherche, et la production peut être liée à la littérature nationale et internationale, montrant une capacité de communication, d'accès, de coopération et de soutien avec ce qui est produit par le monde dans le cadre de l'éducation physique (Coutinho, 2010).

En ce qui concerne la production scientifique dans le Nord, Pereira e Silveira (2019) ont effectué une analyse de la production scientifique des chercheurs de la région du Nord lors de la Réunion nationale de recherche en éducation scientifique et ont indiqué que le Nord a le moins de recherche présent à l'événement. L'étude montre également qu'Amapá n'a effectué aucune présentation et aucun travail dans les éditions 2009, 2011, 2013, 2015 et 2017. Selon l'étude, le plus grand ou le plus petit nombre de productions est lié à la localité du campus universitaire, car elles sont responsables de la plupart des activités scientifiques. Corroborant l'étude susmentionnée, Sidone et coll. (2016) lorsqu'ils ont effectué une analyse de la production d'articles entre les années 2007-2009 des régions du Sud et du Sud-Est, indiquent que celles-ci étaient responsables de plus des trois quarts de la production totale. D'autres régions comme le Nord-Est $15 \%$ et le Midwest et le Nord n'ont pas atteint ensemble $10 \%$ du total national. Outre la concentration des universités dans les régions du Sud et du SudEst, un autre facteur souligné par cette production est la plus grande disponibilité des ressources humaines.

\section{CONCLUSIONS}

Au cours de la maîtrise, la production scientifique sous diverses formes s'est avérée nécessaire au cours du processus de formation. L'union de la théorie avec la pratique de l'écriture, a facilité la production de la thèse qui devrait être présentée à la fin du cours. Grâce au processus d'organisation, la planification des idées a abouti à une 
nouvelle pensée structurée selon les normes de l'académie, mais cela ne se limite pas à cet espace et à ces normes.

Ayant une contribution qui va au-delà de la rédaction scientifique, au cours de ce parcours d'apprentissage a été largement, c'est-à-dire la création d'applications, de sites Web, de livres, d'articles, de méthodologies de pratiques pédagogiques. Toute cette expérience aura un impact positif sur l'avenir en tant que chercheur et professionnel de l'éducation physique.

Dans ce contexte, toute la production au cours de la maîtrise contribue également quantitativement et qualitativement à la production scientifique de la région nord, ce qui a un impact positif sur celle-ci et le programme de maîtrise. En plus de l'importance de la contribution de la création et de la diffusion scientifiques, les productions ont tendance à avoir un impact positif sur l'évaluation et le maintien du programme PROFEPT-PIPT, réalisé par CAPES.

\section{RÉFÉRENCES}

BALBINO, S. I.; URT, S. D. C. Prática Pedagógica em Educação Física para a Educação Integral em Tempo Integral. Pensar a Prática, v. 21, n. 4, p. 775-785, 2018.

CAPES. Ficha de Avaliação - Programas Acadêmicos e Profissionais Área 46: Ensino. Brasília DF, 2020. Disponível em: < https://www.gov.br/capes/pt-br/centraisde-conteudo/FICHA_ENSINO.pdf >. Acesso em: 14 Jun 2021.

COUTINHO, R. X. A influência da Produção Científica nas Práticas de Professores de Educação Física, Ciências e Matemática em Escolas Públicas Municiais de Uruguaiana - RS. 2010. 94p. (Mestrado). Universidade Federal de Santa Maria, Santa Maria RS.

FALASTER, C.; FERREIRA, M. P.; GOUVEA, D. M. R. D. O efeito da publicação científica do orientador na publicação dos seus orientados. Revista de Administração Contemporânea, v. 21, n. 4, p. 458-480, 2017. 
GOOGLEANALYTICS. Google Analytics Aplaticativo Oficial EFE - Pagina Inicial. 2021. Disponível em: < https://analytics.google.com/analytics/web/?authuser=1\#/p265457828/reports/default home >. Acesso em: 14 Jun 2021.

MACHADO, M. F. R. C.; URBANETZ, S. T. Mestrado profissional em educação profissional e tecnológica no Instituto Federal do Paraná: desafios e expectativas. Atos de Pesquisa em Educação, v. 14, n. 2, p. 885-903, 2019.

MAIA, R. T. A importância da disciplina de metodologia científica no desenvolvimento de produções acadêmicas de qualidade no nível superior. Revista Urutágua, v. 14, n. 1, 2008.

PEREIRA, T. Z. M.; SILVEIRA, C. A Produção Acadêmica Da Região Norte: Uma Análise Na Ata Do Xi Encontro Nacional De Pesquisa Em Educação Em Ciências. REAMEC - Rede Amazônica de Educação em Ciências e Matemática, v. 7, n. 2, p. 245-260, 2019.

PLAYCONSOLE. Google Play Console Painel - EFE EduFIsEPT. 2021. Disponível em:

https://play.google.com/console/u/0/developers/4760644030222400117/app/497210 7118591571772/app-dashboard?timespan=thirtyDays\&showKpiMenu=null >. Acesso em: 14 Jun 2021.

RMNC. Revista Multidisciplinar Nucleo do Conhecimento. São Paulo SP, 2021. Disponível em: < https://www.nucleodoconhecimento.com.br/ >. Acesso em: 14 Jun 2021.

ROSSI, A. V. Sobre A Valorização Das Contribuições Da Divulgação Científica No Brasil. In: VOIGT, C. L. (Ed.). O ensino de química. Ponta Grossa PR: Atena Editora, v.1, 2019. cap. 11, p.136-148. 
RSD. Research, Society and Development. Vargem Grande paulista SP, 2021. Disponível em: < https://rsdjournal.org/index.php/rsd >. Acesso em: 14 Jun 2021.

SIDONE, O. J. G.; HADDAD, E. A.; MENA-CHALCO, J. P. A ciência nas regiões brasileiras: evolução da produção e das redes de colaboração científica. Transinformação, v. 28, n. 1, p. 15-32, 2016.

URBANETZ, S. T.; CASSIANO, E. L.; BETTONI, V. O Mestrado Profissional Em Educação Profissional E Tecnológica-Profept-E O Significado Dessa Oferta De Formação Em Pós Graduação No Brasil. Movimento-revista de educação, v. 7, n. 14, 2020.

VIANA, V. N.; DIAS, C. A. G. D. M. Educação Física e EPT - Temáticas em Educação Profissional e Tecnológica (EPT). Macapá AP: 2020. 45 p. ISBN 97865-86069-90-7. em:

https://www.nucleodoconhecimento.com.br/livros/wp-content/uploads/2020/09/livroeducacao-Fisica-e-EPT-educacao-profissional-e-tecnologica-2020.pdf > .

\section{Physical Education and PTE - Topics in Professional and Technological} Education (PTE). Macapá AP: 2020b. 45 p. ISBN 978-65-86069-89-1. Disponível em: < https://www.nucleodoconhecimento.com.br/livros/en/education/physicaleducation-and-pte $>$.

VIANA, V. N. et al. Quadras de esportes em condições de uso adequado no Brasil: influência no ensino-aprendizagem nas aulas de Educação Física (2015). Research, Society and Development, v. 9, n. 8, p. e239985704, 2020. Disponível em: < https://rsdjournal.org/index.php/rsd/article/view/5704/4727 >.

VIANA, V. N. et al. Manuel de pratiques en éducation physique en éducation professionnelle et technologique utilisant l'application gratuite EFE - EPTFIS. Revista Científica Multidisciplinar Núcleo do Conhecimento, v. 5, p. 4, 2021d. Disponível 
em: < https://www.nucleodoconhecimento.com.br/education-physics-fr/manuel-depratiques $>$.

- Manuale di pratiche in educazione fisica nell'educazione professionale e tecnologica utilizzando l'applicazione gratuita EFE - EPTFIS. Revista Científica Multidisciplinar Núcleo do Conhecimento, v. 5, n. 4, p. 65-76, 2021e. Disponível em: < https://www.nucleodoconhecimento.com.br/educazione-fisica-it/manuale-dipratiche $>$.

- Handbuch der Praktiken im Sportunterricht im beruflichen und technologischen Unterricht mit der kostenlosen Anwendung EFE - EPTFIS. Revista Científica Multidisciplinar Núcleo do Conhecimento, v. 5, n. 4, p. 6576, 2021f. Disponível em: < https://www.nucleodoconhecimento.com.br/bildungphysik-de/handbuch-der-praktiken >.

. Пособие по практике физического воспитания в профессиональном и технологическом образовании с использованием бесплатного приложения EFE EPTFIS. Revista Científica Multidisciplinar Núcleo do Conhecimento, v. 5, n. 4, p. 65-76, $2021 \mathrm{~g}$.

Disponível em:

https://www.nucleodoconhecimento.com.br/образование-физика-ru/пособие-попрактике >.

VIANA, V. N. et al. Manual De Praticas Em Educação Física Na Educação Profissional E Tecnológica Utilizando O Aplicativo Gratuito EFE - EPTFIS. Revista Científica Multidisciplinar Núcleo do Conhecimento, v. 5, n. 4, p. 65-76, 2021. Disponível em: $\quad<$ https://www.nucleodoconhecimento.com.br/educacao-fisica/manual-depraticas $>$.

. Manual Of Practices In Physical Education In Professional And Technological Education Using The Free Application EFE - EPTFIS. Revista Científica Multidisciplinar Núcleo do Conhecimento, v. 5, n. 4, p. 65-76, 2021b. Disponível em: < https://www.nucleodoconhecimento.com.br/education-physics-en/manual-ofpractices $>$.

RC: 89148

Lien d'accès: https://www.nucleodoconhecimento.com.br/education-fr/etudiant-a-la-maitrise 
- Manual De Prácticas En Educación Física En Educación Profesional Y Tecnológica Utilizando La Aplicación Gratuita EFE - EPTFIS. Revista Científica Multidisciplinar Núcleo do Conhecimento, v. 5, n. 4, p. 65-76, 2021c. Disponível em: < https://www.nucleodoconhecimento.com.br/educacion-fisica-es/manual-depracticas $>$.

YAMAGUCHI, K. K. D. L.; FURTADO, M. A. S. A Escrita Científica Como Aprendizagem Contextualizada: Uma Abordagem Metodológica A Partir De Um Experimento De Mistura De Cores In: VOIGT, C. L. (Ed.). O ensino de química. Ponta Grossa PR: Atena Editora, v.1, 2019. cap. 12, p.149-158.

Déposée: Juin 2021.

Approuvé : Juin 2021. 